\title{
The importance of Post-Mortem Computed Tomography (PMCT) as a Complementary Diagnostic Technique to Traditional Autopsy in a Case of Asphyxia Related-Death
}

\author{
Del Fante Z. ${ }^{1}$, Di Fazio N. ${ }^{1}$, Quattrocchi A. ${ }^{1}$, Fazio V. ${ }^{1}$, De Matteis A. ${ }^{1}$, Arcangeli M. ${ }^{3}$, La Russa R. ${ }^{1,2}$ \\ ${ }^{1}$ Department of Anatomical, Histological, Forensic and Orthopaedic Science, Sapienza University of Rome, \\ Italy, ${ }^{2}$ IRCCS Neuromed, Via Atinense 18, Pozzilli, 86077, Italy, ${ }^{3}$ Department of Life, Health and Environmental \\ Sciences, University of L'Aquila, Italy
}

\begin{abstract}
Although asphyxia related-death is a common occurrence in forensic setting, traditional autopsy with neck dissection is not always sufficient to gain information about potential injuries of the deep structures of the neck.

In this case a 40 years-old white female was found unresponsive on the floor of her living room, in supine position with a widespread brownish area on her neck.

A preliminary PMCT examination showed a fracture line within the hyoid bone.

This finding was confirmed by the autopsy, which revealed soft tissue's haemorrhage of the sternocleidomastoid and sternohyoid muscles and a fracture of the right horn of the hyoid bone.

A 3D reconstruction of the event showed the aggressor standing behind the victim while holding his arm around her neck.

Our study demonstrates that, PMCT represent an effective aid to traditional examination techniques in order to visualize fractures. The former always requires to be associated with autoptic examination.
\end{abstract}

Keywords: asphyxia, CT, autopsy, legal medicine, forensic medicine

\section{Introduction}

Asphyxia related-death is a common occurrence in forensic setting. Indeed, this event can be related to suicide, homicide and accident.

Traditional neck dissection is not always sufficient to gain information about potential injuries of the deep structures of the neck ${ }^{1}$.

\section{Corresponding author:}

\section{Zoe Del Fante}

Department of Anatomical, Histological, Forensic and Orthopaedic Science, Sapienza University of Rome, viale Regina Elena 336, 00161 Roma.

E-mail: zoe.delfante@uniroma1.it, 0649912927
Moreover, in these forensic cases, the external and internal examination aren't useful to obtain any sign which could help to distinguish between different mechanism of asphyctic death.

Since imaging could be better than autopsy in detecting some fractures, intracranial pathologies, and pneumothorax, the introduction of the post-mortem CT (PMCT) has given a great contribute to the field of postmortem diagnostics (e.g. gunshot wounds ${ }^{2}$, sharp and blunt forces, etc. $)^{3,4}$. Because imaging alone cannot diagnose biochemical and toxicological causes, this technique should always be associated with a traditional post-mortem examination ${ }^{5,6}$.

In particular, PMCT with 3D documentation can be very helpful in revealing injuries on the small structures of the neck, that can be masked by soft tissues and 
surrounding bleedings and provides a useful guide for the pathologist in order to choose the right dissecting technique and avoid artifacts or iatrogenic injury to delicate structures, such as hyoid bone or thyroid cartilage ${ }^{1}$.

\section{Case Presentation}

A 40 years-old white female was found unresponsive on the floor of her living room, in supine position. On the neck there was a widespread brownish area, that was more evident on the right side. During the investigation no mean was found that what compatible with an asphyxia dynamic.

Preliminary to the autopsy examination a PMCT was carried out. The 3D reconstructions were rendered using the software OsiriX ${ }^{\circledR}$ on a MacOSX ${ }^{\circledR}$ device. The CT was able to identify different features such as: fracture of the right horn of the hyoid bone with the fracture line running oblique. (Fig. 1).

External examination was carried out showing a well-developed body in an early stage of decomposition (initial skin marbling).

Multiple lesions were found on different sites of the body surface consistent with blunt-force injuries.

Afterwards at the internal examination, a hemorrhage was revealed at the opening of the neck soft tissues. This infiltration was located on the right sternocleidomastoid muscle and right sternohyoid muscle. Neck in situ dissection, as previously detected by the post-mortem $\mathrm{CT}$, showed a fracture of the right horn of the hyoid bone (Fig.2). Further investigations were conducted, comprehensive of histological and toxicological analysis. Whereas histological hyoid's bone specimens highlighted the presence of several markers of vitality, toxicological ones were negative. All these findings were consistent with an asphyxia-related death.

According to these evidences, a 3D reconstruction of the event was performed using the Poser Debut Software. It showed the aggressor standing behind the victim and holding his arm around the victim's neck (Fig.3). After the autopsy, this reconstruction was confirmed by the victim's husband (the aggressor).
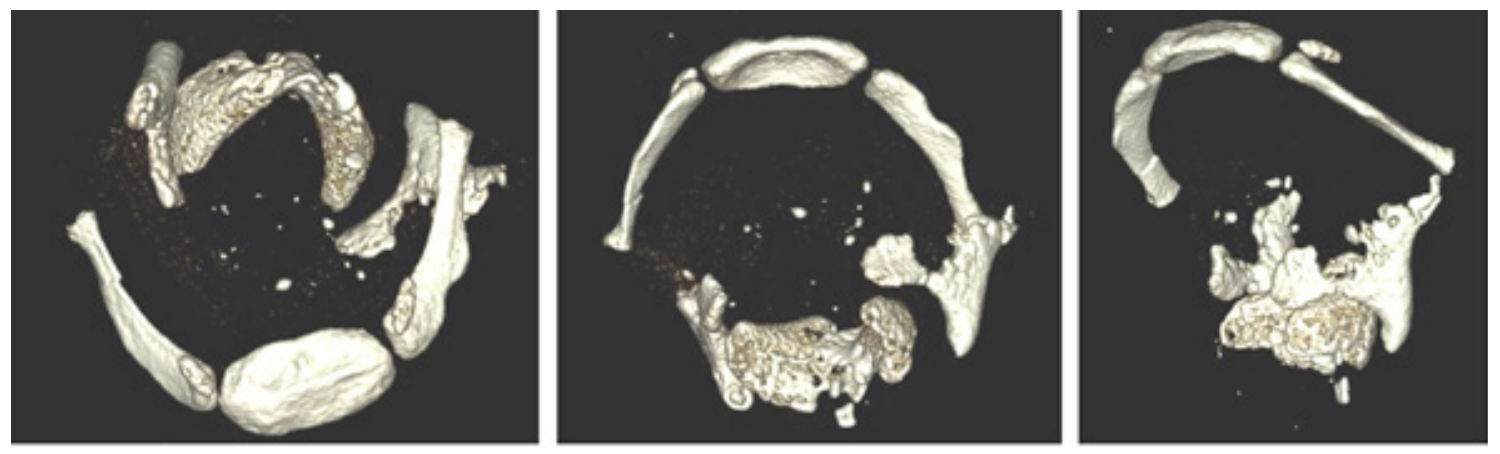

Fig.1: PMCT reconstruction: hyoid fracture was detectable on the right horn with the fracture line running oblique.

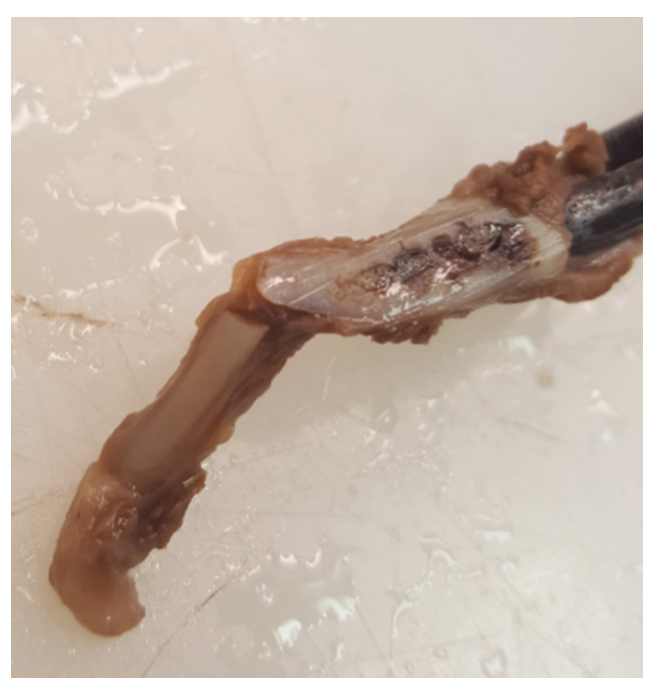

Fig.2: Fracture of the right horn of the hyoid bone after layered in situ dissection of the neck structures. 

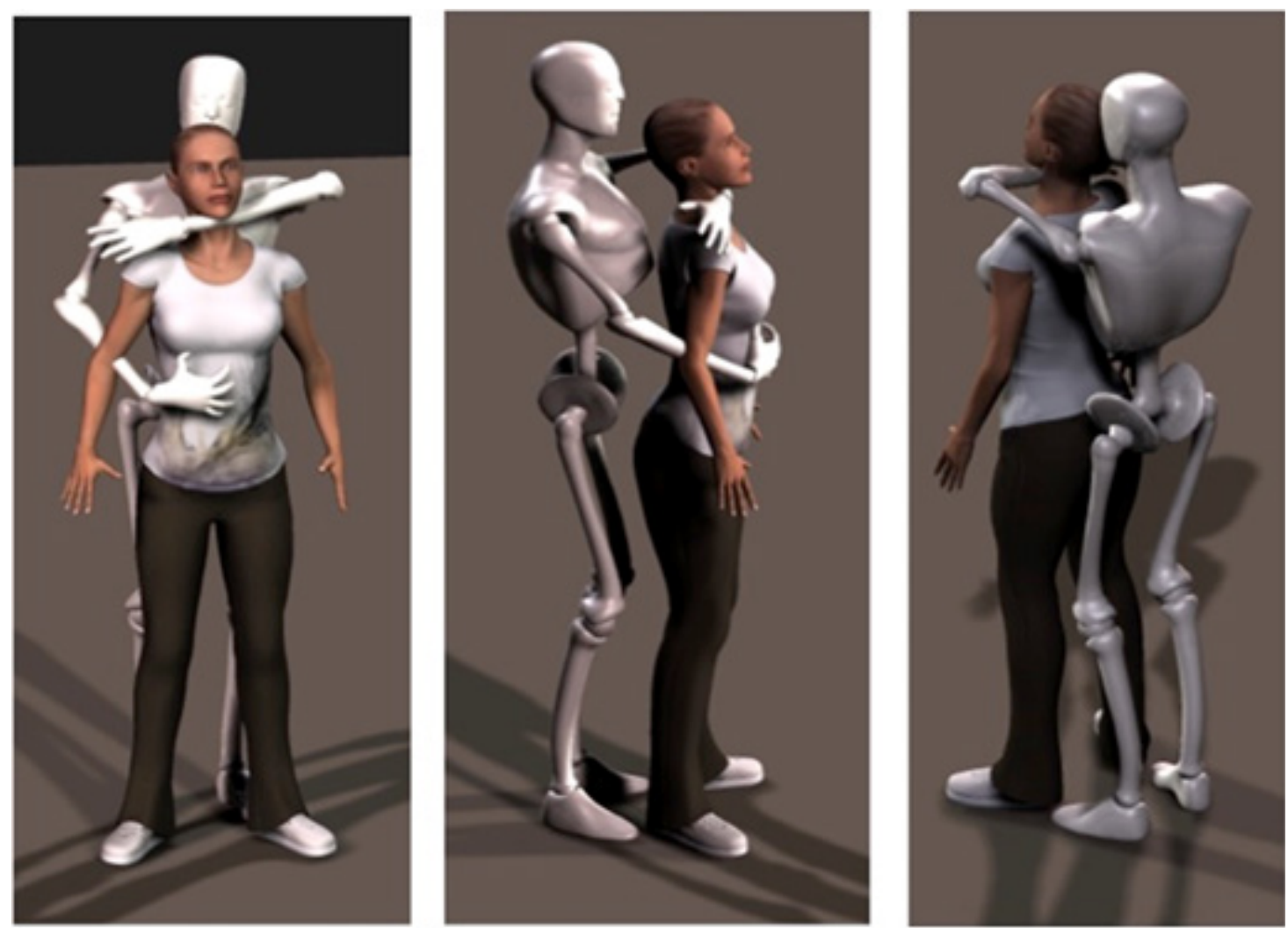

Fig. 3: PMCT 3D reconstruction, using the Poser Debut Software, showed that the aggressor was standing behind the victim while holding his arm around the victim's neck.

\section{Discussion}

In forensic medicine, the term asphyxia describes a situation where there has been a physical obstruction between the mouth and nose to the alveoli, although other 'asphyxial mechanisms' exist, in which there is an inability to utilize oxygen at the cellular level without any physical airway obstruction ${ }^{7}$. Until this day, it has been proved that asphyxias' classification and the definition of various sub-types is not uniform ${ }^{8}$. Asphyxial deaths can be grouped into three categories: suffocation, strangulation and chemical asphyxia. These deaths might be accidental, suicidal or homicidal in manner.

Strangulation is a form of asphyxia which is caused from constriction of the blood vessels and the airways in the neck ${ }^{9,10}$. Furthermore, according to the literature, strangulation can be classified as ligature strangulation (garroting) or manual strangulation (throttling) based on the mechanism used to commit it ${ }^{11,12}$.

Manual strangulation is produced by pressure of the hand, forearm, or other limb against the neck, compressing the internal structures of the neck. The death is due to the occlusion of the blood vessels supplying blood to the brain whereas occlusion of the airway plays a minor role in causing death. Virtually, all cases of manual strangulation are homicide ${ }^{13}$. In the forensic practice, autopsy with dissection of the larynx is the gold-standard to evaluate the presence of specific injuries, which are associated with strangulation. Nevertheless, such features are not always detectable at autopsy ${ }^{14}$.

PMCT is routinely used in forensic death investigations but its role is often limited to the assessment of skeletal injury, gas collections and foreign bodies ${ }^{15,16}$. Thus, an imaging technique such as postmortem TC could be useful to display characteristic findings, including a ligature mark, deviation of the hyoid bone, fracture of the hyoid bone, and fracture of the superior horn of the thyroid cartilage. Even though PMCT may not detect soft tissue injuries in decomposed remains or subtle internal hemorrhages in neck injury, it is more sensitive than autopsy in detecting subtle fractures ${ }^{17}$.

Moreover, the radiological documentation acquired before the autopsy allows the pathologist to choose the right dissecting technique and avoid artifacts or 
iatrogenic injury to delicate structures, such as hyoid bone or thyroid cartilage ${ }^{18}$.

\section{Conclusions}

Our study demonstrates that, in the evaluation of strangulation deaths, PMCT is able to elucidate the essential internal injuries of the bony neck structures and may be equivalent to autopsy-only examinations in the detection of small and difficult to visualize fractures.

In this context, it is of utmost importance for forensic radiologists and forensic pathologists to know how to interpret discrepancies between PMCT and forensic autopsy. The judgment of discrepancies between PMCT and autopsy may influence the interpretation of relevant forensic findings, especially in case of medical liability ${ }^{19}$ and when a forensic case is issued in court.

Nevertheless, PMCT should not be performed alone. In fact, since PMCT remains only a support for forensic medicine practice, it always requires to be associated with autoptic examination.

Specifically, results from our experience and scientific evidence, suggest that PMCT scanning should be proposed in all cases of suspected asphyxia, as the screening approach of first instance to obtain preliminary information useful to rapidly develop the successive autopsy procedure, but it cannot represent an alternative way to the usual autopsy examination ${ }^{20}$.

Ethical Clearance: Taken from Sapienza University of Rome Ethical Commitee

Source of Funding: Self

\section{Conflict of Interest: Nil}

\section{References}

1. Maiese A, Gitto L, dell'Aquila M,Bolino G. When the hidden features become evident: The usefulness of PMCT in a strangulation-related death. Legal Medicine, 2014; 16.6: 364-366.

2. Maiese, A., Gitto, L., De Matteis, A., Panebianco, V., \& Bolino, G. Post mortem computed tomography: useful or unnecessary in gunshot wounds deaths? Two case reports. Legal Medicine, 2014; 16(6), 357-363.

3. Roberts, I. S., et al. Post-mortem imaging as an alternative to autopsy in the diagnosis of adult deaths: a validation study. The Lancet, 2012;
379(9811), 136-142.

4. De Marco, E., et al. Evolution of post-mortem coronary imaging: from selective coronary arteriography to post-mortem CT-angiography and beyond. La radiologia medica, 2018; 1-8.

5. La Russa, R., et al. Postmortem computed tomography angiography (PMCTA) and traditional autopsy in cases of sudden cardiac death due to coronary artery disease: a systematic review and meta-analysis. La radiologia medica, 2019; 124(2), 109-117.

6. Gitto, L., et al. Can post-mortem computed tomography be considered an alternative for autopsy in deaths due to hemopericardium?. Journal of geriatric cardiology: JGC, 2014; 11(4), 363.

7. Payne-James J, Jones R, Karch SB, Manlove J. Simpson's forensic medicine. CRC Press, 2011.

8. Sauvageau A, and Boghossian E. Classification of asphyxia: the need for standardization. Journal of forensic sciences, 2010; $55.5: 1259-1267$.

9. Di Maio VJ, Di Maio D. Asphyxia. In: Geberth VJ, editor. Forensic pathology. Boca Raton, FL: CRC Press; 2001. p. 229-77.

10. Spitz WU. Asphyxia. In: Spitz WU, Spitz DJ, editors. Spitz and Fisher's medicolegal investigation of death: guidelines for the application of pathology to crime investigation. Springfield, IL: Charles C Thomas; 2006. p. 783-845.

11. Oehmichen M, Auer RN, Kçnig HG. Forensic types of ischemia and asphyxia. In: Oehmichen M, editor. Forensic neuropathology and associated neurology. Berlin: Springer-Verlag; 2005. p. 293-313.

12. Yen, K., et al. Strangulation signs: initial correlation of MRI, MSCT, and forensic neck findings. Journal of Magnetic Resonance Imaging: An Official Journal of the International Society for Magnetic Resonance in Medicine, 2005; 22(4), 501-510.

13. Bonanni, E., et al. Femicide in Italy: national scenario and presentation of four cases. MedicoLegal Journal, 2014; 82(1), 32-37.

14. Khokhlov VD. Injuries to the hyoid bone and laryngeal cartilages: effectiveness of different methods of medico-legal investigation. Forensic Sci Int 1997;88(3):173-83.

15. Gitto, L., et al. A scream from the past: a multidisciplinary approach in a concealment of a corpse found mummified. Journal of forensic and 
legal medicine, 2015; 32, 53-58.

16. Maiese, A., et al. The usefulness of post-mortem computed tomography in a crush asphyxia. An excessive enjoyed rave party resulting in a fatal sleep!. Journal of Forensic Radiology and Imaging, 2015; 3(1), 91-95.

17. Decker LA, Hatch GM, Lathrop SL, Nolte KB. The role of postmortem computed tomography in the evaluation of strangulation deaths. Journal of forensic sciences 63.5 (2018): 1401-1405.
18. Bedford PJ. Routine CT scan combined with preliminary examination as a new method in determining the need for autopsy. Forensic Sci Med Pathol. 2012;8:390-4.

19. Marino, V., Mancuso, A., Giugliano, P., Arcangeli, M. A case of recurrent dyspareunia: A matter of medical liability?. Euromediterranean Biomedical Journal, 2018; 13.

20. Pomara C, Fineschi V, Scalzo G, Guglielmi G, Virtopsy versus digital autopsy: virtuous autopsy, Radiol. Med. 2009; 114:1367-1382. 